\title{
Liquid Flow Sensor Based on PVDF in its Beta Phase
}

\author{
J. G. Rocha, G.Minas \\ Dept. Industrial Electronics \\ University of Minho \\ Campus de Azurém, Guimarães, Portugal \\ Email: gerardo@dei.uminho.pt,gminas@dei.uminho.pt
}

\author{
V. Sencadas, S. Lanceros-Mendez \\ Dept. Physics \\ University of Minho \\ Campus de Gualtar, Braga, Portugal \\ Email: vsencadas@fisica.uminho.pt, lanceros@fisica.uminho.pt,
}

\begin{abstract}
This article describes a system for the measurement of liquid or gaseous fluids. The main innovation is that the device is of tubular form and is composed by a single part. It is manufactured from a piezoelectric polymer such as $\beta$-PVDF, in which one electrode in the inside and three electrodes in the outer side are placed. One of the exterior electrodes is excited by an electric voltage, whose frequency, of the order of tens or hundreds of megahertz, produces mechanical oscillations in the area of the pipe in contact with it, producing an ultrasound with the same frequency. The areas of the pipe under the other two electrodes also work as ultrasound transducers, receiving the signal produced by the first one. There are two main methods of reading flows with ultrasound sensors: a method based on the Doppler effect and another method based in the transit time. In both of these methods, they are used the differences of frequency or phase between the emitted and the received signals in order to calculate the speed of the liquid or gas that passes through the pipe. The flow is then calculated by multiplying the speed of the fluid by the area of the section of the pipe. In this project, it was used the technique of the time of transit, where the flux is calculated from the difference between the time that ultrasounds need to travel in the direction of the flow and in the direction opposite to the flow. The main difference between this flow sensor and other ones available in the market is that this sensor is the pipe by itself, becoming simpler, more compact, more robust and cheaper.
\end{abstract}

\section{INTRODUCTION}

Currently, great part of hospital medical equipments is automatic, being its interaction with computers and respective databases made in a total transparent form. Among the processes that are still manual, it is the one that gave origin to this article. It consists in the measurement of the amount of urine expelled by patients lying in bed. Currently this urine is stored in a bag, and it is necessary a nurse to register manually its amount several times a day.

The main goal of the project is to measure automatically the amounts of urine expelled for each patient and send the measured values directly to a computer that will deal with the information in the adequate form. Thus, one of the biggest challenges of the project consists in the fact that the flow sensor to be used needs to be very compact and cheap, in order to place a unit in each urine bag, which is discarded each time it is filled up.

The flow sensor consists in a device of tubular form manufactured from a piezoelectric polymer, such as poli(fluorine vinilidine) (PVDF) in the $\beta$ phase [1], in which there are placed three electrodes: one working as ultrasound emitter and two as receivers. The central electrode is excited by an electric voltage whose frequency must be in the order of the hundreds of megahertz, causes a mechanical oscillation in the area of the pipe that is in contact with it, producing ultrasounds. The areas of the pipe under the two lateral electrodes will work as ultrasound receptors, receiving the signals that come from the first one. The speed of the ultrasound that propagates in the liquid medium is affected by the speed of the liquid by itself. From the ultrasound speed difference, it is possible to determine the speed of the liquid in the interior of the pipe and then calculate its volume. The main difference between this flow sensor of and other ones available in the market is that the sensor is the pipe by itself, becoming simpler, more compact, more robust and cheaper.

Other applications for the flow sensor (with small adjustments or alterations) can be considered, namely in domestic water counters, petrol pumps, etc.

This article is organized as follow:

- Section II describes the state of the art of the flow sensors. Some patents that show the most recent advances in this area are presented. The theoretical basis of the flow sensors based on ultrasound waves is also explained in this section.

- Section III presents the techniques used in the fabrication of the sensor, section IV presents some preliminary experimental results and finally section $\mathrm{V}$ presents some conclusions and comments.

\section{BACKGROUND}

In order to detect a flow that crosses a pipe, it must be used a flow meter, whose working principle can be based, between other methods, on ultrasound waves. In this in case, there are used acoustic waves with frequencies larger than $20 \mathrm{kHz}$. Depending on geometry, the transducers that will emit and receive the ultrasonic waves can be in direct contact with the measured fluid or can be isolated from it by a material that makes the acoustic coupling between the transducer and the fluid.

The main advantages of the ultrasonic flow sensors relatively to others that use some other working principles, are the following: 
- There is no blockage to the circulation of the fluid, and then there are no zones of pressure fall in the interior of the pipe.

- There are no moving parts, not being necessary its maintenance.

- It can be used to measure corrosive fluids or semifluids as mud or cement.

Basically, there are two main methods to design ultrasonic flow sensors: The first one is based in the Doppler effect of and the second one is based on the time of transit of ultrasounds.

Usually, either in the Doppler Effect method or in the time of transit one, there are used one or several piezoelectric transducers (piezocrystals or piezoceramics) coupled in some way to the pipe where fluid circulates.

\section{A. Flow sensor based on the Doppler Effect}

The Doppler Effect indicates that the frequencies of the sound waves depend on the movement of the emitter and the receiver transducers relatively to the propagation medium. To use this effect in the measure of liquid flows in a pipe, a transducer emits ultrasounds, at a frequency of hundreds of $\mathrm{kHz}$, through the flow. Some particles or air bubbles suspended in the liquid fluid reflect the ultrasounds back to a second transducer (Fig. 1).

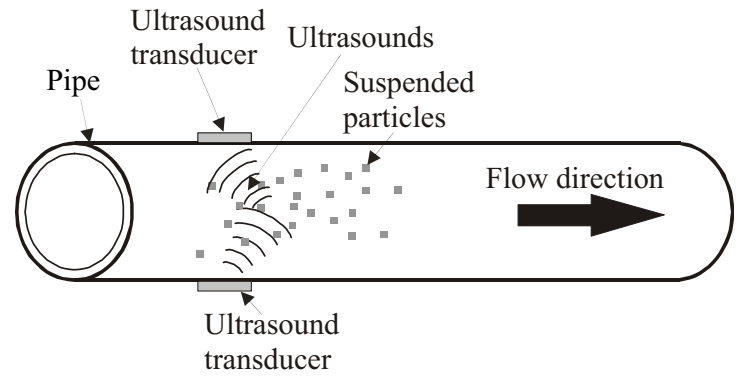

Fig. 1: Sensor based on the Doppler Effect with two ultrasound transducers. In order to work properly, it is necessary that the liquid have particles or air bubbles suspended on it. The ultrasound transducer emits ultrasounds, at a frequency of hundreds of $\mathrm{kHz}$, through the flow. The particles suspended in the liquid reflect the ultrasounds to the second transducer that will receive them.

A simpler arrangement can be obtained with only one transducer, which can emit and receive the sound waves. In this case it is necessary that they are pulsed, that is, the transducer sends a pulse and waits for the echo (Fig. 2). Then, a second pulse is sent and thus successively.

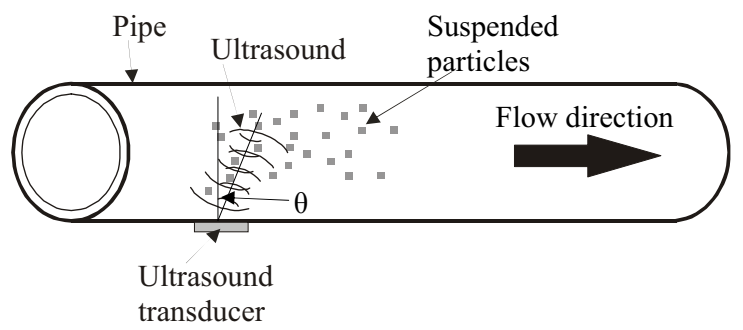

Fig. 2: Sensor based on the Doppler Effect with only one ultrasound transducer. The ultrasound transducer emits ultrasounds through the flow. The particles suspended in the liquid reflect the ultrasounds back to the transducer that will receive them.
The movement of suspended particles or air bubbles modifies the frequency of the reflected beam in accordance with the Doppler Effect. The frequency shift is proportional to the speed of these particles and as a consequence, to the speed of the liquid that crosses the pipe. The basic equations that govern this phenomenon are:

$$
\Delta f=2 f_{T} \sin \theta \frac{v}{c}
$$

where $\Delta f$ is the Doppler frequency shift, $f_{T}$ is the operating frequency of the ultrasound emitter, $\theta$ is the angle with that ultrasound enters in the flow, $v$ is the flux velocity and $c$ is the velocity of sound in the fluid, when it is at rest. According to Snell's law:

$$
\frac{\sin \theta_{T}}{c_{T}}=\frac{\sin \theta}{c}
$$

where $\theta_{T}$ is the angle between the ultrasound propagation direction and the flow direction and $c_{T}$ is the ultrasound propagation velocity in the material that constitutes the emitter.

From equations (1) and (2) one can obtain:

$$
v=\frac{\Delta f}{2 f_{T}} \frac{c_{T}}{\sin \theta_{T}}=K \Delta f,
$$

where:

$$
K=\frac{c_{T}}{2 f_{T} \sin \theta_{T}}
$$

Equation (3) shows that the speed of the fluid is proportional to the Doppler frequency shift. Once the speed is obtained, the flow is calculated by multiplying it by the area of the pipe. The main drawback of the Doppler effect for measuring flows is that it only works properly if there are air bubbles or particles suspended in the fluid.

The patent document US2005/0209793 [2] describes a flow sensor based on the Doppler Effect, where only one ultrasonic transducer is connected to the pipe. An algorithm of correction of the quantization error of is also described.

The document JP2004108946 [3] describes a flow sensor based on the Doppler Effect that additionally measures the real speed of propagation of the sound in the liquid in question. In this case, they are used four ultrasonic transducers: one to measure the Doppler frequency shift and the other three to measure the speed of the sound in the liquid circulating in the pipe.

The document JP2005241437 [4] describes a flow sensor based on the Doppler Effect, where it is used one technique to reduce the influence of the resultant acoustic noise of the reflection between the exterior face of the pipe and the transducer and the reverberation emitted by the transducer itself. In this case, it is used an ultrasonic transducer mounted in the exterior of the pipe.

Documents US2005011279 [5] and US2005245827 [6] describe a flow sensor based on the Doppler Effect and a system of reading it. In this case, it is used an ultrasonic transducer placed in the exterior of the pipe.

Document WO2005083371 [7] describes a flow sensor based on the Doppler Effect that uses two ultrasonic 
transducers: one to emit the pulses and other to receive the produced echoes. The transducers are placed in the exterior of the pipe.

\section{B. Flow sensor based on the Time of Transit of the Ultrasounds}

Beyond the sensors based on the Doppler Effect, there exists another type of ultrasound flow sensors: the ones based on the time of transit of ultrasounds. The flow sensors based on the time of transit, as the name indicates, measure the difference between the time delays necessary for the ultrasounds to travel a certain distance in the flow direction and in the contrary direction.

In this case, they are also usually used piezoelectric transducers (piezocrystals or piezoceramics) connected in some way to a pipe in which the flow circulates, in order to measure the flow.

The time of transit in the direction of the flow can be calculated from:

$$
T_{1}=\frac{L}{c+V \cos \theta},
$$

where $L$ is the distance between the transducers and $\theta$ is the angle between the flow direction and the imaginary line that connects the two transducers. In the same way, the transit time in the direction opposite to the flow can be calculated from:

$$
T_{2}=\frac{L}{c-V \cos \theta}
$$

Equations (5) and (6) can be solved simultaneously in order to $c$ and $v$, obtaining the ultrasound propagation velocity in the fluid and the fluid velocity:

$$
\begin{gathered}
c=\frac{L\left(T_{1}+T_{2}\right)}{2 T_{1} T_{2}} \\
v=\frac{L\left(T_{2}-T_{1}\right)}{2 T_{1} T_{2} \cos \theta}
\end{gathered}
$$

Once again, to calculate the flow, it is enough to multiply the fluid velocity by the area of the pipe.

There are several ways to implement the flow sensor based on the time of transit of ultrasounds. These implementations can be based either in two, three or four transducers.

Fig. 3 shows a flow sensor based on the time of transit, where there are used only two ultrasonic transducers. Each one of the transducers works alternately as emitter or receptor. The direct time of transit, $T_{1}$, is measured when the bottom left sensor works as emitter and the top right one works as receptor. In the same way, the inverse time of transit, $T_{2}$, is measured when the top right sensor works as emitter and the bottom left one works as receptor.

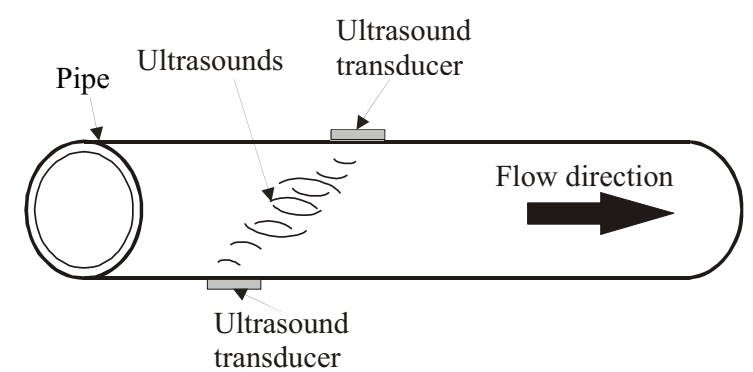

Fig. 3: Flow sensor based on the time of transit with two ultrasonic transducers. Each one of the transducers works alternately as emitter or receptor. The direct time of transit is measured when the bottom left sensor works as emitter and the top right one works as receptor. The inverse time of transit is measured when the top right sensor works as emitter and the bottom left one works as receptor.

Fig. 4 shows a flow sensor based on the time of transit of the ultrasounds, where three transducers are used. In this case, the top transducer works as ultrasound emitter and the two bottom transducers work as ultrasound receptors. $T_{1}$ will be the time that the ultrasound takes in reaching the right bottom transducer. By the same way, $T_{2}$ will be the time that the ultrasound takes in reaching the left bottom transducer.

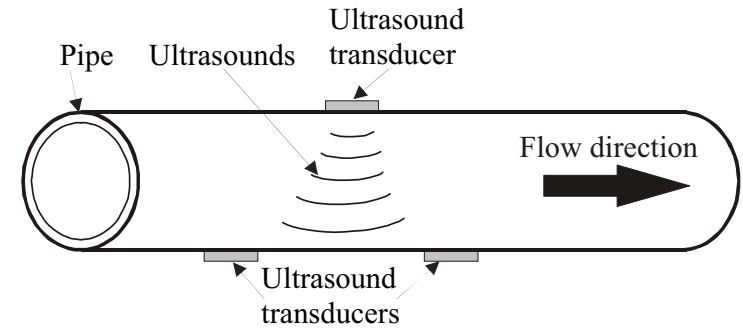

Fig. 4: Flow sensor based on the time of transit of the ultrasounds with three transducers. In this case, the top transducer works as ultrasound emitter and the two bottom transducers work as ultrasound receptors.

Fig. 5 shows a flow sensor based on the time of transit, where four ultrasound transducers are used. In this case, each one of the transducers, at its time, works as emitter, and the transducer at the opposite side works as receptor.

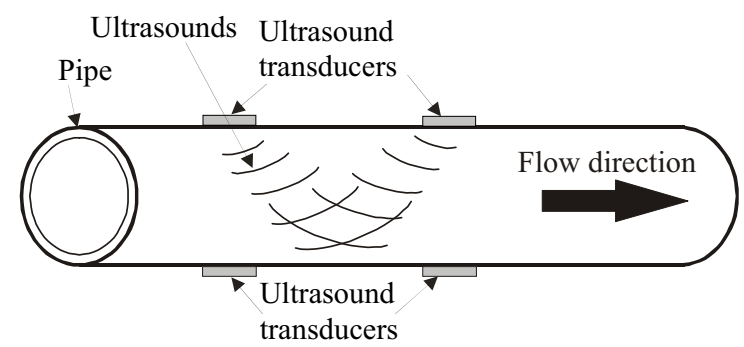

Fig. 5: flow sensor based on the time of transit with four ultrasound transducers. Each one of the transducers, at its time, works as emitter, and the transducer at the opposite side works as receptor.

As an example, when the transducer placed at the bottom left side works as emitter, the transducer placed at the top right side works as receptor. In this case, $T_{1}$ is calculated from the average of two time values: the first one is the time of transit when the bottom left side transducer works as emitter (and the top right side transducer works as receptor) and the second one 
is the time of transit when the top left side transducer works as emitter (and the bottom right side transducer works as receptor). The time of transit $T_{2}$ is calculated in a similar way by interchanging the emitters by the receptors and vice-versa.

Document EP1211488A2 [8] describes a flow sensor based on the time of transit and uses two ultrasonic transducers connected to a pipe folded in $U$ form. The two transducers work alternately as ultrasonic emitter/receptor.

Document GB2300265 [9] describes a flow sensor based on the time of transit and uses four transducers of ultrasounds and one temperature sensor connected to the pipe. From the four ultrasonic transducers, two determine the transit time, the third is used to measure the speed of the sound in the fluid and the fourth measures the thickness of the walls of the pipe.

Document GB2400439 [10] describes a flow sensor based on the time of transit and uses two piezoelectric transducers in a ring shape, placed in two cuts made in the pipe.

Of the known and previously presented state-of-the-art, no technology relates the use of a piezoelectric polymer pipe similar to our work.

\section{Ultrasound transducer based on Beta-PVDF}

The relationships that describe the piezoelectric behavior can be obtained from the thermodynamic principles [12]. By taking into account the electrical and elastic properties of the material, in a general form, they are the following $[13,14]$ :

$$
\begin{aligned}
& D=d+\varepsilon^{\sigma} E \\
& S=s^{E} \sigma+d E
\end{aligned}
$$

Equation (9) describes the direct piezoelectric effect and equation (10) describes its reverse. The stress $\sigma$ and the electric field $E$ were chosen as independent variables, while the deformation $S$ and the electric displacement $D$ are dependent variables. The parameters $s^{E}, \varepsilon^{\sigma}$ and $d$ are respectively the elasticity of the material at a constant electric field, the permittivity at a constant mechanical stress and the piezoelectric constant.

According to the description of the piezoelectric behavior, an ultrasonic transducer, in its basic form, can be fabricated from a $\beta$-PVDF film [1], in witch two metallic contacts are placed, as shown in fig. 6 .

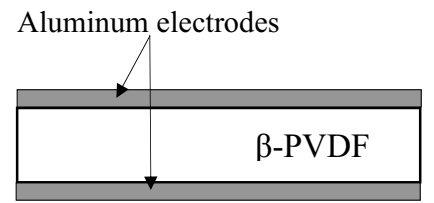

Fig. 6: Structure of a ultrasound transducer: A $\beta$-PVDF film where two metallic electrodes are placed.

An applied electrical voltage between the metallic electrodes increases the electric field inside the $\beta$-PVDF, causing its deformation. To produce an ultrasound, it is then enough to apply an alternated voltage to the electrodes, whose frequency is in the ultrasonic range,

\section{SENSOR DESIGN AND FABRICATION}

In our flow sensor, instead of a normal polymeric pipe, with ultrasonic transducers attached to it, we use a pipe fabricated from a piezoelectric polymer $(\beta-\mathrm{PVDF})$. This is the main difference between our sensor and the other ones available in the market. We decided to build the flow sensor based on the time of transit with one central electrode that works as emitter and two lateral electrodes, which work as receptors. Its working principle is similar to the one of fig. 4 .

\section{A. Fabrication of the pipe}

In any one of the two types of flow sensors, the pipe can be manufactured from a piezoelectric polymer, where it is placed one electrode of conducting material (aluminum, for example) in its interior and one or more electrodes of conducting material in its exterior (Fig. 7). These electrodes and the piezoelectric polymer form ultrasound transducers.

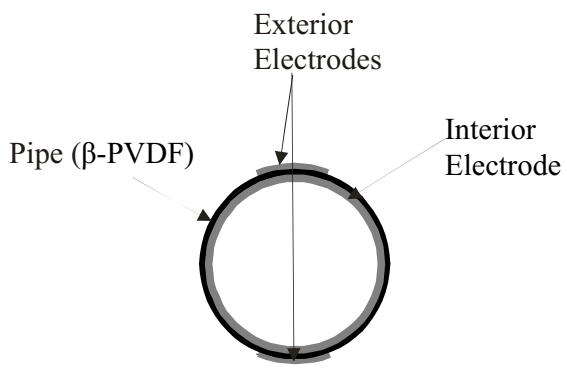

Fig. 7: Structure of the sensor. The pipe is manufactured from a piezoelectric polymer ( $\beta$-PVDF), where it is placed one electrode of aluminum, in its interior and one or more electrodes of aluminum in its exterior.

The pipe is obtained from a poli(fluoreto of vinilideno) (PVDF) polymer by tubular extrusion from a PVDF resin. The extruded material is stretched until a the final length is larger than four times the initial one at a temperature lower than $100^{\circ} \mathrm{C}$. This stage originates a transformation of the non-polar phase ( $\alpha$ phase) into an electrically active phase ( $\beta$ phase) [15]. After this procedure, it is applied an intense electric field, in the order of some kilovolts, in the direction of the thickness, allowing the optimization of the electroactive properties of the material. After this stage, the material is subjected to the deposition of the electrical contacts.

\section{B. Readout electronics}

The readout electronic of the sensor can be divided into two parts: the electronics for actuation of the ultrasonic transducer working as an emitter and the read-out electronics of the receptors. The first one consists basically in an oscillator and the second one consists in two phase comparators.

The electronics for actuation of the transducer working as an emitter is based in a Colpitts oscillator that produces a sinusoidal wave with $12 \mathrm{~V}$ of amplitude and a frequency of $130 \mathrm{kHz}$.

The readout electronics for the signals received by the two other transducers need to be amplified. For this amplification, an instrumentation amplifier was chosen. In order to determine 
the transit times $T_{1}$ and $T_{2}$, the phases of the signals read by the receptors must be compared to the phase of the emitted signal. As the frequencies of the signals are equal, this comparison of the phases can be performed with a simple logic OR-Exclusive circuit.

\section{PRELIMINARY EXPERIMENTAL RESUlts}

Fig. 8 shows the waveforms applied to the emitter and read directly from one of the receptors. In this case, the pipe is filled with air at rest. There exist a phase shift between the two signals that correspond to the time that the ultrasound needs to travel the distance between the transducers.

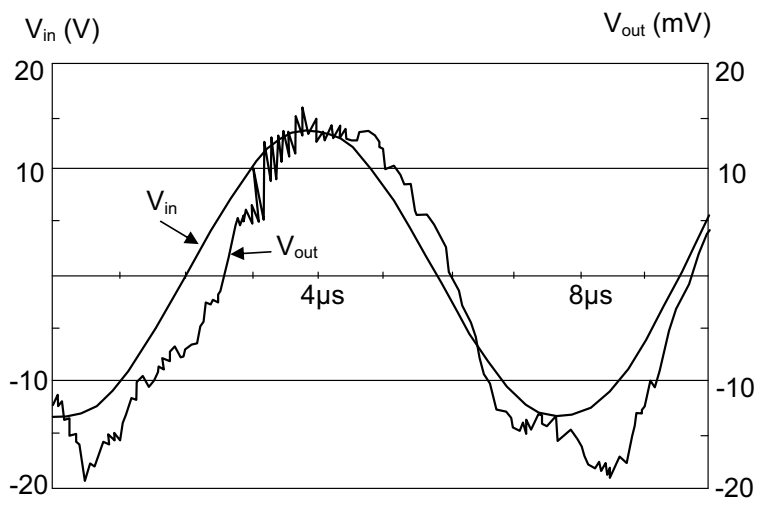

Fig. 8: Waveforms applied to the ultrasound emitter and received at one of the receptors. The pipe is filled with air at rest.

Fig. 9 shows the same waveforms, but in this case the air is forced to circulate in the pipe against the direction of propagation of the ultrasound. Comparatively to fig. 8, the phase shift increases.

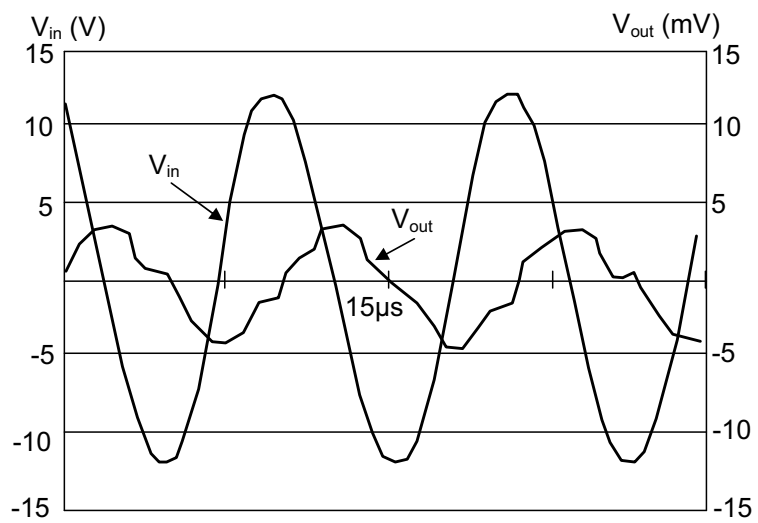

Fig. 9: Waveforms applied to the emitter and read from the receptor when some air is forced to circulate inside the pipe. In this case, the phase shift will increase once the direction of the air is opposite to the direction of propagation of the ultrasounds.

Fig. 10 shows the same waveforms, but in this case, the pipe is filled with water at rest, which is a better ultrasound conducting material than air.

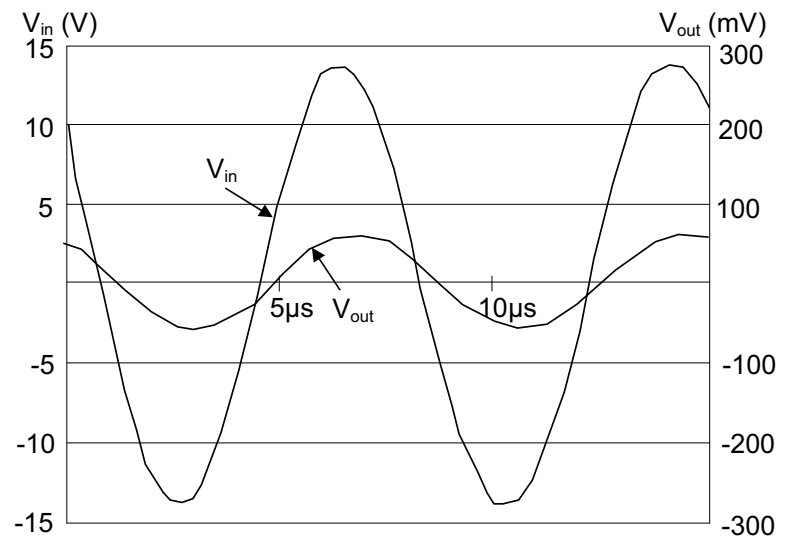

Fig. 10: Waveforms applied to the ultrasound emitter and received at one of the receptors. The pipe is filled with water at rest.

Fig. 11 shows the same waveforms, but in this case the water is forced to circulate in the pipe in the direction of propagation of the ultrasound. Comparatively to fig. 10, the phase shift decreases.

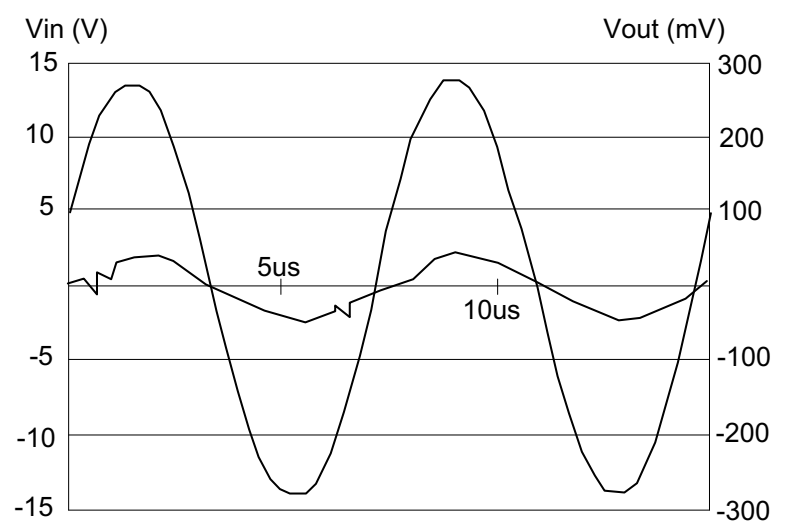

Fig. 11: Waveforms applied to the emitter and read from the receptor when some water is forced to circulate inside the pipe. In this case, the phase shift will decrease once the direction of the water and the direction of propagation of the ultrasound are coincident.

Beyond the expected differences in phase, these preliminary experiments showed the also expected fact that the ultrasound travel faster and with less losses in the liquid media than in air. The amplitude of the wave at the receptors is approximately 10 times higher when the flow is liquid.

\section{CONCLUSIONS}

A liquid or gas sensor based in a single tube performed from piezoelectric polymer has been presented. The sensor works on the principle of the time of transit of the ultrasounds. The present sensor shows several advantages with respect to the sensors currently used and/or patented: it is simple, robust and compact. It can be fabricated in basically three steps and allows diameters below one millimeter to several centimeters. As present and future work, we will continue to test and characterize the new flow sensor. 


\section{ACKNOWLEDGEMENTS}

The authors thank the Portuguese Foundation for Science and Technology - FCT (Grant POCI/CTM/59425/2004) for financial support. They also thank to Ricardo Lameiro and Heloisa Costa for their help building sensor. V. Sencadas thanks to FCT for the PhD Grant (SFRH/BD/16543/2004)

\section{REFERENCES}

[1] Sencadas, V., Gregorio Jr., R., Lanceros-Mendez, S., Processing and characterisation of a novel nonporous poly(vinilidene fluoride) films in the $\beta$ phase. J. Non-Crystalline Solids, 352, 2226, 2006.

[2] Yamada Kazuyuki, Ohmuro Yoshinori, Hirayama Noritomo, Kishiro Masami, "Doppler Ultrasonic Flowmeter, And Processor And Method Thereof With Quantization Error Correction," Patent US2005209793, 2005.

[3] Mori Harutsugu, Tezuka Kenichi, Futaki Shoichiro, Tezuka Hideaki, "Doppler Type Ultrasonic Flowmeter, And Flow Rate Measuring Method And Computer Program Using Doppler Type Ultrasonic Flowmeter," Patent JP2004108946, 2004

[4] Hirayama Noritomo, Yamamoto Toshihiro, Yao Hironobu, Onodera Takuya, "Doppler Type Ultrasonic Flowmeter," Patent JP2005241437, 2005.
[5] Takeda Yasushi, Mori Michitsugu, "Doppler Ultrasonic Flowmeter," Patent US2005011279, 2005.

[6] Takeda Yasushi, Mori Michitsugu, "Doppler Type Ultrasonic Flowmeter, Flow Rate Measuring Method Using Doppler Type Ultrasonic Flowmeter And Flow Rate Measuring Program Used In This Doppler Type Ultrasonic Flowmeter," Patent US2005245827, 2005.

[7] Ohmuro Yoshinori, Yao Hironobu, Yamamoto Toshihiro, "Doppler Type Ultrasonic Flowmeter," Patent WO2005083371, 2005.

[9] Kobayashi Tamotsu, Fujii Toru, Shigeta Kunikazu, "Transit-time difference type ultrasonic flowmeter,"Patent EP1211488, 2002

[10] Carrington Andrew Brian; Rydin Philip Ake Mansson, "Flowmeter," Patent GB2300265, 1996

[11] Sanderson M. L., Hemp J.," Ultrasonic flowmeter with flush mounting ring shaped transducers for propagating axisymmetric waves along a flowtube," Patent GB2400439, 2004.

[12] W. P. Mason, "Physical Acoustics and the Properties of Solids," D. Van Nostrand Co. Inc, Princeton, NJ, 1958

[13] Czekaj, Dionizy, "Technology, Properties and Applications of PZT thin films," Katowic, 2002, 24- 28.

[14] Ikeda, Takuro, "Fundamentals of Piezoelectricity," Oxford Science Publications, Oxford University Press, 1996.

[15] V. Sencadas S. Lanceros-Mendez; R. Gregorio-Filho; A.S. Pouzada "ato $b$ transformation on PVDF films obtained by uniaxial stretch" Materials Science Forum, Volume 514 - 516, p. 872, 2006 